\title{
RA-GSMC Design for a SISO Servo-system with Nonlinear Uncertainty
}

\author{
SUN Xiu Juan ${ }^{1, a}$ and WANG Chuan Jiang ${ }^{1,2} b^{*}$ \\ ${ }^{1}$ College of Electrical Engineering and Automation, SDUST, Qingdao, 266590, China \\ ${ }^{2}$ School of Control Science and Engineering, Shandong University, Jinan, 250061, China \\ a sun8sd@163.com, ${ }^{b}$ cxjwang@163.com \\ * corresponding author
}

Keywords: Nonlinearity, Sliding mode control, Servo-system

Abstract. Considering the parameter uncertainties, model inaccuracies and external disturbances in the servo-system, it is difficult to achieve an ideal performance of the conventional sliding mode controller (SMC), so we designed a robust adaptive global sliding mode controller (RA-GSMC) by constructing a global sliding mode function and an adaptive law, which can enable the system to reach the sliding mode surface at the very beginning and move along the surface until the end. Moreover, the adaptive law estimates the system lump uncertainty so as to amend the control law output, which enables the perfect control of the system. Simulation demonstrates the validity of the control scheme.

\section{Introduction}

It is well known that SMC control scheme is widely used in control fields owing to its effectiveness in dealing with system parameter uncertainties, nonlinearities and external disturbances. Generally, SMC is designed to make all the state variables move toward a pre-specified sliding surface, once the system reaches the destined sliding surface, it will move along this surface till the system stable state.

The conventional SMC scheme includes reaching phase and sliding mode phase, and the former can reduce the robustness of the system. Therefore, how to reduce the reaching phase is considered most important. In [1], the reaching phase was shortened by proposing a piecewise-constant moving switching surface, which made the designed SMC system insensitive to the parameter uncertainties and external disturbances in the reaching phase. Reference [2] described the system tracking errors by getting rid of the time-dependent parameter from the original tracking error, which eliminated the reaching phase and made the tracking error become zero from the original state. A time-varying switching plane is adopted to eliminate the reaching phase for nonlinear systems with parameter uncertainty and external disturbances [3], and uses a type-2 fuzzy system to estimate the unknown system factors [3, 4]. References [5, 6] designed the SMC using an adaptive switching gain to maintain the system response, and alleviated any effects caused by disturbances and uncertainties. Furthermore, super-twisting SMC design methods were studied for the linear and time invariant uncertain system by adjusting variable gains of the algorithm and updating the slope of the sliding surface via fuzzy logic schemes $[7,8]$. As a result, the robustness is guaranteed and the dynamic performance is improved.

In order to eliminate the reaching phase in conventional SMCs, the global sliding mode controller (GSMC) has became a prevailing technology being researched, which ensures the system has a sliding mode in the entire process [9-12]. REA-GSMC was designed by defining a sliding mode function as an exponential type, and finishing the tracking control for a two-link robot manipulator [9]. A generalized GSMC based on REA-GSMC was then devised, which enhanced the robustness and performance in decreasing the bad influences caused by the uncertainties [10]. Reference [12] brought forward a method of the global sliding mode function with an integral expression, and gave detailed design steps of the GSMC. With the continuous development of GSMC, the combination of GSMC with other methods arose. Moreover, [11] carried out the design of discrete GSMC for the discrete SISO system, the discrete reaching law is provided, and the stability is analyzed.

In the GSMC system, the reaching phase has been removed by the global sliding mode function, which enables the system to arrive at the sliding mode condition from the beginning. However, for 
systems with parameter uncertainties, nonlinearity and external disturbances, which decrease the robustness of the control system, it is advisable to design a controller which has the ability to estimate the system lumped uncertainty. So, as for the SISO nonlinear uncertain servo-system, we design a robust adaptive-GSMC scheme.

\section{System Description}

Consider the following n-th order SISO nonlinear uncertain system:

$$
x^{f}(t)=[A+\Delta A(t)] X(t)+[B+\Delta B(t)] U(t)+f(t)
$$

Where, the state parameter vector $X(t) \in \mathrm{R}^{n}$, control vector $U(t) \in \mathrm{R}$, and the external disturbance $f(t) \in \mathrm{R}^{n}, A \in \mathrm{R}^{n \times n}$. Meanwhile, the vectors $\Delta A(t)$ and $\Delta B(t)$ are parameter perturbation vectors, and $f(t)$ is the system nonlinearities.

Suppose the vectors $\Delta A(t), \Delta B(t)$ and $f(t)$ are continuous functions, and the matching condition is $\Delta A(t)=B D(t), \Delta B(t)=B H(t)$ and $f(t)=B L(t)$.

Where, $D(t) \in \mathrm{R}^{n}, L(t) \in \mathrm{R}$ and $H(t) \in \mathrm{R}$, while $|H(t)|<1$. Let $E(t)$ be the lumped uncertainty including parameter perturbation, nonlinearity and external disturbances, which is bounded and unknown.

So, (1) of the system can be written in the following form:

$$
\mathcal{X}(t)=[A+\Delta A(t)] X(t)+[B+\Delta B(t)] U(t)+f(t)=A X(t)+B(U(t)+D(t) X(t)+H(t) U(t)+L(t))=A X(t)+B(U(t)+E(t))
$$

Where, $E(t)=D(t) X(t)+H(t) U(t)+L(t)$.

\section{RA-GSMC Design}

Sliding Mode Function Design. According to the system, the defined tracking error is shown as:

$$
X_{e}(t)=R(t)-X(t)
$$

where, $R(t) \in \mathrm{R}^{n}$ is the target movement vector. Suppose the initial tracking error is $X_{e}(0)$, we can get the global sliding mode function as the following equation:

$$
s(t)=C X_{e}(t)-C M(t) X_{e}(0)
$$

where, $\left.C f_{c_{1}}, c_{2}, \mathrm{~L}, c_{n-1}, 1\right]$, is a sliding mode parameter vector, and $c_{i}>0, i=1,2, \mathrm{~L}, n-1$, meanwhile, we define the sliding mode moving parameter vector as:

$$
M(t)=\operatorname{diag}\left[\exp \left(-\beta_{1} t\right) \quad, \mathrm{L} \quad, \quad \exp \left(-\beta_{n} t\right)\right]
$$

where $\operatorname{Re}\left(\beta_{i}\right)>0, i=1,2, \mathrm{~L}, n$. When $t=0$, then $s(0)=0$, and when $t \rightarrow \infty$, owing to $\lim _{t \rightarrow \infty} X_{c}(t)=0$, therefore, the equation $\lim _{t \rightarrow \infty} s(t)=\lim _{t \rightarrow \infty}\left[C X_{e}(t)-C M(t) X_{e}(t)\right]=0$, can be obtained. That means the system attains the sliding surface from the original state without the reaching movement, and maintains it until the end. Meanwhile, the favorable dynamic performance can be achieved by vectors $C$ and $M(t)$.

Controller Design. In order to decrease or eliminate the chattering of the system, it is advisable to identify the bounded-unknown uncertainty of the system, so, an adaptive law was designed, which can adjust the control law automatically according to the uncertainty. Let $\hat{E}(t)$ be defined as the evaluation of system uncertainty $E(t)$. Thus, we get the following control law:

$$
U(t)=(C B)^{-1}\left[C R(t)-C A X(t)-C N \notin(t) X_{e}(0)-C B \hat{E}(t)+\sigma s(t)\right]
$$

where, $U_{e q}(t)=(C B)^{-1}\left[C\left(\left\{t(t)-C A X(t)-C\left(\left\{(t) X_{e}(0)\right]\right.\right.\right.\right.$, is an equivalent control item obtained by the equation $\$(t)=0$ without considering the parameter perturbation, system nonlinearity and external disturbances, which can make the system move along the sliding surface until the anticipated target is reached. Moreover, the $\sigma>0$, is a given constant, and the adaptive law is described by

$$
\stackrel{\varepsilon}{\varepsilon}(t)=-\lambda^{-1} C B s(t) \text {. }
$$

where, $\lambda$ is a given positive constant.

According to the designed control law, let the Lyapunov function candidate be defined as:

$$
V=\frac{1}{2} s^{2}(t)+\frac{1}{2} \lambda \mathscr{E}^{\circ}(t)
$$

where, $\mathscr{E}(q)=\hat{E}(t)-E(t)$ is the identification error of lumped uncertainty. Then the time derivative of $V$ is

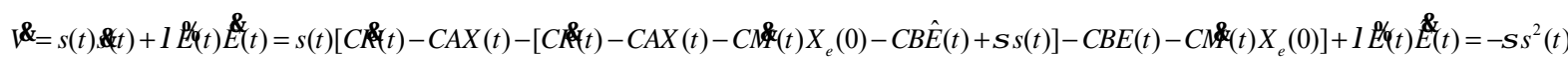


So, we can obtain the result $r^{\alpha}=-\sigma s^{2} \leq 0$, the system is asymptotically stable controlled by the designed RA-GSMC algorithm.

In order to illuminate the improved performance of the designed RA-GSMC, we contrast it against the conventional GSMC shown in (10).

$$
U(t)=(C B)^{-1}\left[C R(t)-C A X(t)-C n £(t) X_{e}(0)\right]+\rho \operatorname{sgn}(s(t))
$$

where,

$$
\operatorname{sgn}(s(t))=\left\{\begin{array}{ll}
1, & \text { if } s(t)>0 \\
0, & \text { if } s(t)=0 \\
-1, & \text { if } s(t)<0
\end{array},\right.
$$

and $\rho$ is the upper limit of $E(t), \rho \geq|E(t)|>0$, the $\rho \operatorname{sgn}(s(t))$ part is a switching item, which can generate the sliding mode of the system. Moreover, the $(C B)^{-1}\left[C\left(R(t)-C A X(t)-C \&(t) X_{e}(0)\right]\right.$ is an equivalent control item.

Let the Lyapunov function candidate be $V_{G S M C}=\frac{1}{2} s^{2}(t)$, then the time derivative of $V_{G S M C}$ is

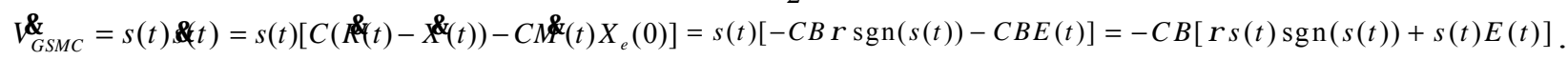

Owing to $C B>0, \rho \geq|E(t)|>0$ and $s(t) \operatorname{sgn}(s(t)) \geq 0$, so $\downarrow_{G S M C} \leq 0$, the system is asymptotically stable.

\section{Experiment}

Servo-system Description. Considering that the electro-hydraulic servo-system is a typical nonlinear system with parameter perturbation and external disturbances shown as Fig. 1, we can define the system input signal vector and the system state vector as $R(t)=\left[r_{1} ; r_{2} ; r_{3}\right]$ and $X(t)=\left[x_{1} ; x_{2} ; x_{3}\right]$ respectively, meanwhile, $r_{2}=\$, r_{3}=\kappa_{1}$, and $x_{2}=\ldots, x_{3}=$.

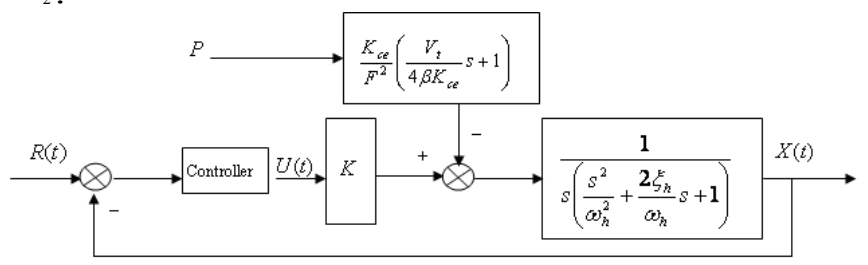

Fig.1 Diagram of the control system

Where, $F=5.41 \times 10^{-3} \mathrm{~m}^{2}$ is the plunger area of the hydro-cylinder, $K_{c c}$ is the flow-pressure coefficient of proportional valve, $K_{c e}=5.16 \times 10^{-11} \mathrm{~m}^{5} / \mathrm{N} \cdot \mathrm{s} . \beta=7 \times 10^{8} \mathrm{~N} / \mathrm{m}^{2}$ is the equivalent bulk modulus of hydraulic oil and tube wall, $V_{t}=1.4 \times 10^{-3} \mathrm{~m}^{3}$ is the total volume of pipeline and oil reservoir on both sides of hydro-cylinder, $m=100 \mathrm{~kg}$ represents the load mass. Moreover, $P$ means the load force, $K$ is the velocity gain of the valve-controlled hydro-cylinder, and $\omega_{h}, \xi_{h}$ are nature frequency and relative damping coefficient of hydro-cylinder respectively, meanwhile, $\omega_{h}=765 \mathrm{rad} / \mathrm{s}, \xi_{h}=0.07$. So the system state matrix is shown as:

$$
\mathcal{W}(t)=\left[\begin{array}{l}
\& \\
\hat{\alpha} \\
\delta
\end{array}\right]=\left[\begin{array}{ccc}
0 & 1 & 0 \\
0 & 0 & 1 \\
0 & -a_{2} & -a_{1}
\end{array}\right]\left[\begin{array}{c}
x_{1} \\
x_{2} \\
x_{3}
\end{array}\right]+\left[\begin{array}{l}
0 \\
0 \\
b
\end{array}\right] U(t)+\left[\begin{array}{l}
0 \\
0 \\
f_{L}
\end{array}\right]=\left[\begin{array}{ccc}
0 & 1 & 0 \\
0 & 0 & 1 \\
0 & -\left(a_{20}+\Delta a_{20}\right) & -\left(a_{10}+\Delta a_{10}\right)
\end{array}\right]\left[\begin{array}{c}
x_{1} \\
x_{2} \\
x_{3}
\end{array}\right]+\left[\begin{array}{c}
0 \\
0 \\
b_{0}+\Delta b_{0}
\end{array}\right] U(t)+\left[\begin{array}{c}
0 \\
0 \\
f_{L}
\end{array}\right]=\left[\begin{array}{ccc}
0 & 1 & 0 \\
0 & 0 & 1 \\
0 & -a_{20} & -a_{10}
\end{array}\right]\left[\begin{array}{c}
x_{1} \\
x_{2} \\
x_{3}
\end{array}\right]+\left[\begin{array}{c}
0 \\
0 \\
b_{0}
\end{array}\right](U(t)+E(t))
$$

where $a_{10}, a_{20}$ and $b_{0}$ are the system nominal parameters, $\Delta a_{10}, \Delta a_{20}$ and $\Delta b_{0}$ are system perturbations, and $a_{10}=2 \xi_{n} \omega_{h}=107.1, a_{20}=\omega_{h}^{2}=585225, b_{0}=K \omega_{h}^{2}=114665$. Meanwhile, $E(t)$ is the lumped uncertainty, and $f_{L}$ is the disturbance can be written as:

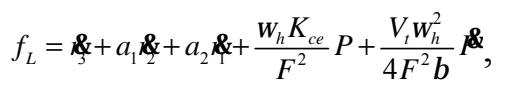

which is a continuous function, and is the change rate of load force $P$.

Simulation. According to the above nonlinear- uncertain servo-system, the tracking error is shown as

$$
X_{e}(t)=R(t)-X(t)=\left[\begin{array}{lll}
r_{1}-x_{1}, & r_{2}-x_{2}, & r_{3}-x_{3}
\end{array}\right]^{T} .
$$

We carry out the simulation by adopting the designed sliding mode function shown in (4) and the control law shown in (6). The sliding mode parameter vector $C$ is designed by using the pole assignment method, where the vectors $C=[200,20,1]$, and $\beta=[10,20,10]$.

Meanwhile, we designed the parameters $\sigma=50$, and $\lambda=25000$. Suppose the initial state is $X(t)=[0 ; 0 ; 0]$. The step response of the RA-GSMC system are shown in Fig. 2 in the circumstance of $E(t)=0.5 \sin (2 \pi t)$. 


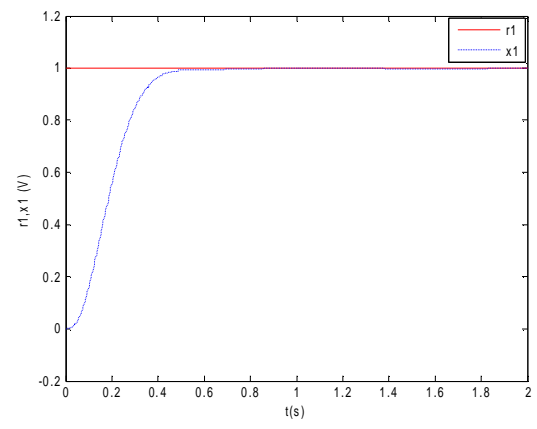

(a) Step response

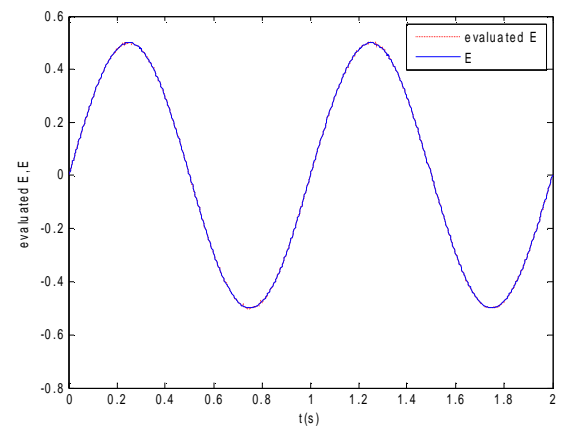

(c) Trajectories of $\mathrm{E}$ and evaluated $\mathrm{E}$

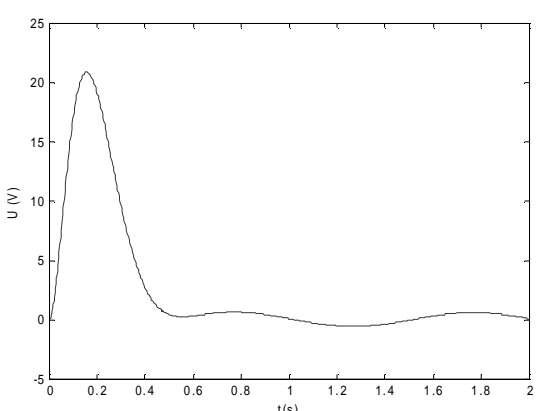

(b) Control law

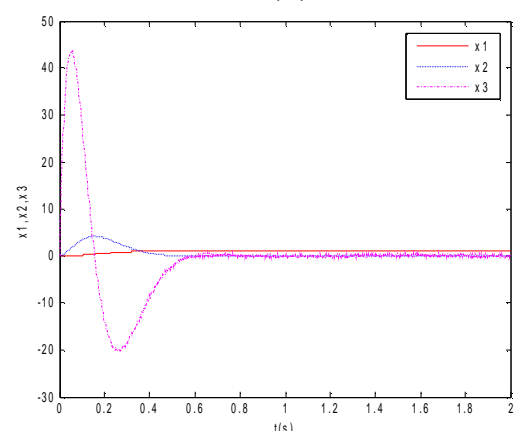

(d) Variables of the system

Fig.2 Step response results of RA-GSMC

Fig.2 (a) to (d) show that the RA- GSMC algorithm have good control effects and dynamic performance, and can finish the exact estimation about the system uncertainty caused by the parameter perturbations and external load disturbances.Then, for contrast, the simulation results of the conventional GSMC are shown in Fig. 3 , where $\rho=0.5$, is the maximum of system uncertainty.

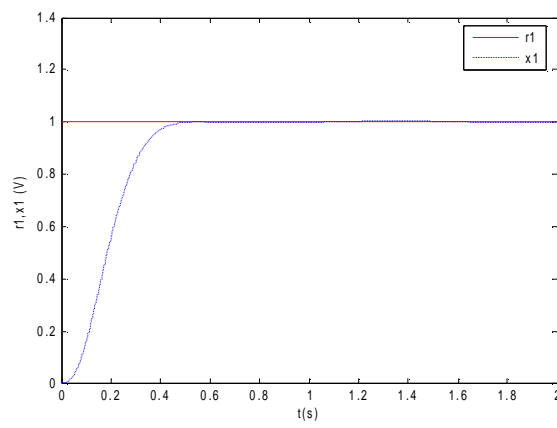

(a) Step response

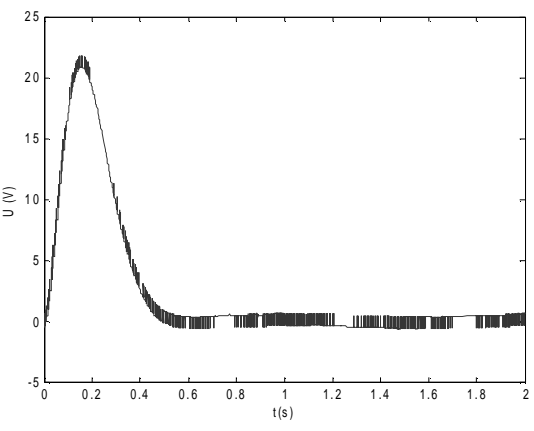

(b) Control law

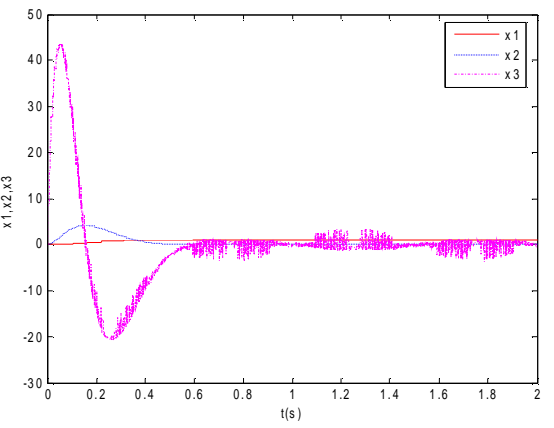

(c) Variables of the system

Fig.3 Step response results of conventional GSMC

From Fig. 3 (a) to (c), it is clearly that the conventional GSMC system has chattering which can be alleviated in the RA-GSMC method. In order to further indicate the efficiency of RA-GSMC, $E(t)$ is supposed a rectangle wave type signal, and the simulation results are shown in Fig.4. 


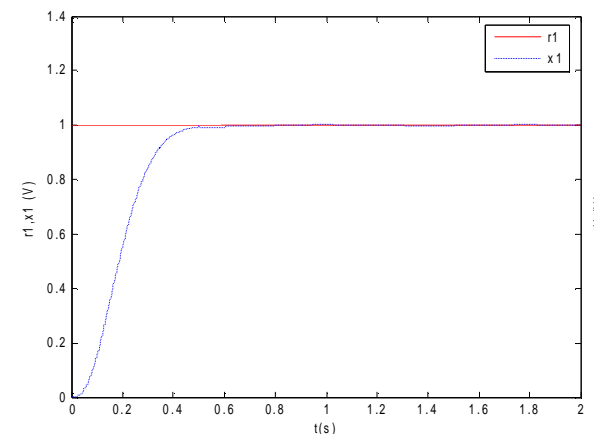

(a) Step response

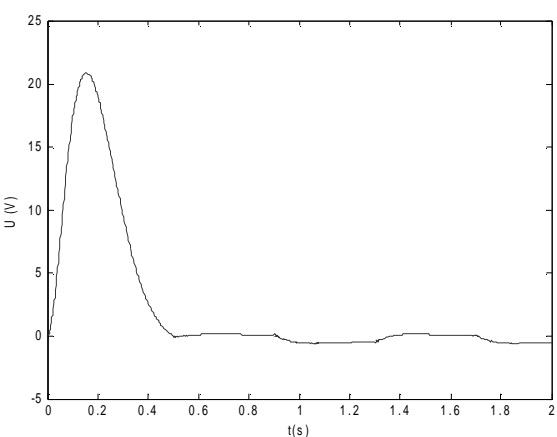

(b) Control law

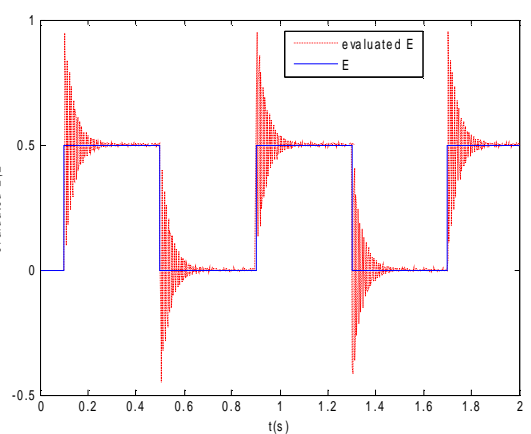

(c) Trajectories of $\mathrm{E}$ and evaluated $\mathrm{E}$

Fig.4 Step response results of RA-GSMC while $E(t)$ is a rectangle wave signal

Fig.4 (a) to (c) show that the RA-GSMC system has better performance to quick track the bounded and nonlinear system uncertainties, and $\hat{E}(t)$ can track and keep pace with $E(t)$ within about 0.18 seconds, meanwhile, the control law is smooth and the position tracking is perfect.

\section{Conclusion}

This paper proposed a RA-GSMC for a SISO servo-system with bounded nonlinear uncertainty. The global sliding mode function can make the system on the sliding surface from the original state, and the adaptive law estimates the system uncertainties accurately, which indicates the system has ideal dynamic performances. The simulation results verify the validity of the designed control method.

\section{Acknowledgements}

This work was financially supported by the Shandong Science and Technology Development Plan (2013GSF11858), and the Qingdao Science and Technology Development Plan (13-1-3-121-nsh).

\section{References}

[1] S. B. Choi, C. C. Cheong, D.W. Park, Moving switching surfaces for robust control of second order variable structure systems, Int. J. Control. 58(1993) 229-245.

[2] T. H. Chang, Y. Hurmuzlu, Sliding control without reaching phase and its application to bipedal locomotion, ASME J. Dyn. Syst., Meas., Control. 115(1993) 447-455.

[3] A. Al-khazraji, N. Essounbouli, A. Hamzaoui, F. Nollet, J. Zaytoon, Type-2 fuzzy sliding mode control without reaching phase for nonlinear system, Eng. Appl. Artif. Intell. 24(2011) 23-28.

[4] M. Benbrahim, N. Essounbouli, A. Hamzaoui, A. Betta, Adaptive type-2 fuzzy sliding mode controller for SISO nonlinear systems subject to actuator faults, Int. J. Autom. Comput. 10(2013) 335-342.

[5] K. Jamoussi, L. Chrifi-Alaoui, H. Benderradji, A. El Hajjaji, M. Ouali, Robust sliding mode control using adaptive switching gain for induction motors, Int. J. Autom. Comput. 10(2013) 303-311.

[6] Z. Chen, B. L. Cong, X. D. Liu, Switching gain reduction in adaptive sliding mode control for rigid spacecraft attitude maneuvers, Mathematical Problems in Engineering. 2013(2013) 1-9.

[7] T. Gonzalez, J. A. Moreno, L. Fridman, Variable gain super-twisting sliding mode control, IEEE Trans. Autom. Control. 57(2012) 2100-2105.

[8] A. Kareem, M. F. Azeem, A novel adaptive super-twisting sliding mode controller with a single input-single output fuzzy logic control based moving sliding surface, Int. J. Control. Autom. 6(2013) 183-198. 
[9] Y. S. Lu, J. S. Chen, Design of a global sliding mode controller for robot manipulator with robust tracking capability, Proceedings of the National Science Council-Part A: Physical Science and Engineering. 18(1994) 463-476.

[10] Y. S. Lu, C. W. Chiu, Global sliding-mode control with generalized sliding dynamics, Asia J. Control. 11(2009) 449-456.

[11] Y. Ni, J. P. Xu, Study of discrete global-sliding mode control for switching DC-DC converter, J. Circuits, Syst. Comput. 20(2011) 1197-1209.

[12] L. Yu, M. Q. Zhang, S. M. Fei, Non-linear adaptive sliding mode switching control with average dwell-time, Int. J. Syst. Sci. 44(2013) 471-478. 